\title{
Draft genome sequences of two Bifidobacterium sp. from the honey bee (Apis mellifera)
}

\author{
Kirk E Anderson ${ }^{1,2^{*}}$, Andreas Johansson ${ }^{1,3}$, Tim H Sheehan ${ }^{1,4}$, Brendon M Mott ${ }^{1}$, Vanessa Corby-Harris ${ }^{1}$, \\ Laurel Johnstone ${ }^{5}$, Ryan Sprissler ${ }^{5}$ and William Fitz ${ }^{2,5}$
}

\begin{abstract}
Background: Widely considered probiotic organisms, Bifidobacteria are common inhabitants of the alimentary tract of animals including insects. Bifidobacteria identified from the honey bee are found in larval guts and throughout the alimentary tract, but attain their greatest abundance in the adult hind gut. To further understand the role of Bifidobacteria in honey bees, we sequenced two strains of Bifidobacterium cultured from different alimentary tract environments and life stages.

Results: Reflecting an oxygen-rich niche, both strains possessed catalase, peroxidase, superoxide-dismutase and respiratory chain enzymes indicative of oxidative metabolism. The strains show markedly different carbohydrate processing capabilities, with one possessing auxiliary and key enzymes of the Entner-Doudoroff pathway.

Conclusions: As a result of long term co-evolution, honey bee associated Bifidobacterium may harbor considerable strain diversity reflecting adaptation to a variety of different honey bee microenvironments and hive-mediated vertical transmission between generations.
\end{abstract}

Keywords: Bifidobacterium, Probioiotic, Apis mellifera, Honey bee, Crop, Respiratory metabolic pathway, ROS tolerance

\section{Background}

Bifidobacterium are common animal commensals, used as probiotics, and widely considered important to host metabolism [1]. Most are strict anaerobes, but Bifidobacterium asteroides PRL22011, isolated from the honey bee hindgut, was recently sequenced and found to carry genes for oxidative respiration and protection from reactive oxygen species [2]. Moreover, a phylogenomic analysis from the same study suggests that Bifidobacteria associated with the honey bee is of ancient origin relative to Bifidobacteria in mammals. Culture based results and 454 amplicon sequencing demonstrate that Bifdobacteria can be found throughout the alimentary tract but reside primarily in the hind gut of honey bees [3-5]. To more thoroughly characterize the breadth of strain diversity and metabolic potential in honey bee Bifidobacterium, we sequenced two additional

\footnotetext{
* Correspondence: kirk.anderson@ars.usda.gov

${ }^{1}$ Carl Hayden Bee Research Center, USDA-ARS, Tucson AZ 85719, USA

${ }^{2}$ Department of Entomology, University of Arizona, Tucson AZ 85721, USA

Full list of author information is available at the end of the article
}

strains sampled from different honey bee alimentary tract microenvironments.

The honey bee hive is composed of a variety of nutrient rich microenvironments generated by exposed, typically continuous larval rearing and substantial food storage. These dynamic and highly variable niches support microbial communities specific to the hive environment, and are governed by a variety of biotic and abiotic factors including $\mathrm{pH}$, acidity, oxygen exposure, hygroscopy, and honey bee secreted enzymes [6,7]. Following the transition of the honey bee from the larval to adult stage, the transmission of Bifidobacteria and other core bacteria to the gut of the newly emerged adult is seemingly accomplished via the hive environment and/or trophallaxis with older siblings [3,4]. Both of these routes expose Bifidobacteria to extremes of $\mathrm{pH}$ and oxygen found in the foregut and hive environments.

\section{Methods}

\section{Bacterial culture}

Bifidobacterium strain A11 was isolated from the gut of a third instar larvae sampled from a feral Africanized 
Table 1 Metrics associated with sequencing and assembly of two strains of Bifidobacteria

\begin{tabular}{cccccccc}
\hline Strain & Total reads & Total bases & Total contigs & N50 contig size & Total contig length & Genome coverage & \%GC content \\
\hline 7101 & 262,222 & $60,733,017$ & 19 & 524,826 & $2,117,598$ & $24 X$ & 59.74 \\
A11 & 286,838 & $70,227,442$ & 51 & 223,528 & $2,180,865$ & $27 X$ & 60.10 \\
\hline
\end{tabular}

honey bee colony near Oracle, AZ [7]. Strain 7101 was isolated from the foregut (crop) of an adult nurse worker bee sampled from a managed European colony at the Carl Hayden Bee Research Center in Tucson, AZ [3]. Bacterial strains were isolated using De Man Rosaga Sharp (MRS) media under aerobic (strain A11) or microaerophilic (strain 7101 ) conditions at $35^{\circ} \mathrm{C}$. Bacterial isolates were picked and regrown in liquid MRS media to attain enough DNA for sequencing.

\section{Nucleic acid isolation}

A $300 \mu \mathrm{l}$ aliquot of each MRS culture sample was centrifuged at 12,000 $\mathrm{g}$ for $5 \mathrm{~min}$. After decanting supernatant, bacterial pellets were lyzed at $37^{\circ} \mathrm{C}$ for $1 \mathrm{~h}$ with $300 \mu \mathrm{L}$ of lysozyme lysis buffer $(100 \mathrm{mM} \mathrm{NaCl}, 500 \mathrm{mM}$ Tris [pH 8.0], lysozyme $10 \mathrm{mg} / \mathrm{ml})$. We then added $200 \mu \mathrm{l}$ of SDS lysis buffer (100 mM NaCl, $500 \mathrm{mM}$ Tris [pH 8.0], $10 \%$ [wt./vol.] SDS) and vortexed. After incubation at $65^{\circ} \mathrm{C}$ for $10 \mathrm{~min}$, the mixture was centrifuged at $12,000 \mathrm{~g}$ for $5 \mathrm{~min}$. The supernatant was transferred to another microcentrifuge tube. Protein was removed by adding $500 \mu \mathrm{l}$ of chloroform/isoamyl alcohol (24:1), vortexing for $5 \mathrm{~s}$, incubating at $4^{\circ} \mathrm{C}$ for $5 \mathrm{~min}$, and centrifuging at $12,000 \mathrm{~g}$ for $5 \mathrm{~min}$. The upper solution was precipitated by adding a $0.5 \mathrm{vol}$. of $7.5 \mathrm{M}$ ammonium acetate and a 1.0 vol. of isopropanol. After incubation at $-20^{\circ} \mathrm{C}$ for $15 \mathrm{~min}$, DNA was pelleted at $12,000 \mathrm{~g}$ for $10 \mathrm{~min}$ and washed three times with $75 \%$ ethanol. DNA pellets were air dried, then resuspended in $100 \mu \mathrm{l}$ of $10 \mathrm{mM}$ Tris, $\mathrm{pH}$ 8.0.

\section{Library preparation and sequencing}

We quantified DNA using PicoGreen, nebulized $600 \mathrm{ng}$ of each sample and prepared the libraries according to the Rapid Library Preparation protocol, using Multiplex Identifiers RLMID8 and RLMID10 for strain A11 and 7101 respectively. Genome sequences were obtained at the University of Arizona Genomics Core using Roche 454 GS pyrosequencing and a whole genome shotgun strategy.

\section{Read quality assessment}

Sequencing reads were assembled de novo using Roche 454 software, Newbler version 2.6 with default settings (Table 1). We used the RAST server [8] and accompanying SEED database for gene prediction and annotation (Table 2). Genome sequence submission to NCBI resulted in the reannotation of the assemblies according to the standards of the Prokaryotic Genome Automatic Annotation Pipeline (PGAAP).

\section{Quality assurance}

Throughout many steps of the process, Sanger sequencing of the 16S rRNA gene confirmed that both isolates were pure and $>99 \%$ similar to previously submitted Bifidobacterium sequences. B. asteroides PRL2011 differed

\section{Table 2 Categories of functional roles (subsystems) of Bifidobacterium strains 7101 and $\mathrm{A} 11$ based on RAST} subsystem annotation

\begin{tabular}{|c|c|c|}
\hline \multirow[t]{2}{*}{ Subsystem features } & \multicolumn{2}{|c|}{$\begin{array}{l}\text { Subsystem feature } \\
\text { counts by strain }\end{array}$} \\
\hline & 7101 & A11 \\
\hline Cofactors, vitamins, prosthetic groups, pigments & 71 & 70 \\
\hline Cell wall and capsule & 42 & 53 \\
\hline Virulence, disease and defense & 12 & 11 \\
\hline Potassium metabolism & 2 & 2 \\
\hline Photosynthesis & 0 & 0 \\
\hline Miscellaneous & 6 & 6 \\
\hline Phages, prophages, transposable elements, plasmids & 0 & 2 \\
\hline Membrane transport & 28 & 12 \\
\hline Iron acquisition and metabolism & 0 & 0 \\
\hline RNA metabolism & 59 & 56 \\
\hline Nucleosides and nucleotides & 61 & 59 \\
\hline Protein metabolism & 157 & 158 \\
\hline Cell division and cell cycle & 19 & 19 \\
\hline Motility and chemotaxis & 0 & 4 \\
\hline Regulation and cell signaling & 26 & 24 \\
\hline Secondary metabolism & 0 & 0 \\
\hline DNA metabolism & 60 & 62 \\
\hline Regulons & 0 & 0 \\
\hline Fatty Acids, lipids, and isoprenoids & 31 & 29 \\
\hline Nitrogen metabolism & 7 & 7 \\
\hline Dormancy and sporulation & 1 & 1 \\
\hline Respiration & 21 & 21 \\
\hline Stress response & 41 & 41 \\
\hline Metabolism of aromatic compounds & 3 & 3 \\
\hline Amino acids and derivatives & 155 & 158 \\
\hline Sulfur metabolism & 13 & 12 \\
\hline Phosphorus metabolism & 22 & 22 \\
\hline Carbohydrates & 183 & 235 \\
\hline
\end{tabular}


from each strain at 10 of $147316 \mathrm{~S}$ rDNA nucleotide positions. Strain A11 and 7101 differed from one another at 4 of 1473 nucleotide positions.

\section{Initial findings}

Both strains lack the glycolytic enzyme phosphofructokinase-1, but possess the enzymatic marker indicative of genus Bifidobacterium: fructose-6-phosphate phosphoketolase, historically referred to as the "bifid shunt" [9]. Unlike typical Bifidobacterium, and as described previously for honey bee associated Bifidobacterium [2], both strains also possess oxidative respiratory pathways, and genes that cope with reactive oxygen species, including catalase, peroxidase and superoxide-dismutase. Consistent with co-evolution in and around harsh osmoregulatory conditions [6,7], the transmembrane channel aquaporin $\mathrm{Z}$ was present in both genomes. This protein is highly stable, facilitates both rapid and long term osmoregulation, and resists denaturing due to heat, detergent, or extremes of $\mathrm{pH}$.

Absent in strain 7101, strain A11 possesses genes for chemotaxis, and the Entner-Doudoroff pathway. Found in many pathogenic bacteria [10], strain A11 has the dTDP-rhamnose biosynthetic pathway, which may play a part in cell wall integrity, growth and/or host interaction. Lacking in strain A11, strain 7101 contains 5 different EFC class transporters dedicated to the importation of vitamins, and 4 CRISPR-associated proteins predicted to provide immunity against genetic parasites.

\section{Future directions}

The extent of strain diversity and associated function of Bifidobacteria in honeybees remains unclear. Identification of the metabolic potential of different strains provides information on the predicted survival of unique strains in different gut and hive microenvironments. Comparative transcriptomics under different environmental conditions may elucidate candidate strains for probiotic treatment, a viable alternative or complement to traditional treatments typically applied to honey bee colonies.

\section{Availability of supporting data}

The draft genome sequences of Bifidobacterium strain A11 and strain 7101 were deposited in DDBJ/EMBL/ GenBank under the accessions AWUO00000000 and AWUN00000000 respectively.

Competing interests

The authors declare no competing interests.

\section{Authors' contributions}

KEA planned the experiments and wrote the paper. AJ, BMM and THS cultured, isolated, and identified the bacteria. WF and VCH extracted the DNA and provided genome metrics. $L$ and RS processed the genome assemblies. All authors read and approved the final manuscript.

\section{Acknowledgments}

The first author thanks his uncle "Buzzy", University of Idaho microbiologist Dr. Guy R. Anderson, a connoisseur of nitrogen-fixing soil bacteria. We thank Colleen Ramsower and Heather Issar at the University of Arizona BIO5 genomics institute. This work was supported by the ARS-USDA, an equal opportunity employer and provider.

\section{Author details}

${ }^{1}$ Carl Hayden Bee Research Center, USDA-ARS, Tucson AZ 85719, USA ${ }^{2}$ Department of Entomology, University of Arizona, Tucson AZ 85721, USA. ${ }^{3}$ National Oceanography Centre, University of Southampton, Southampton, SO14 3ZH, UK. ${ }^{4}$ Department of Microbiology, University of Arizona, Tucson AZ 85721, USA. ${ }^{5}$ Bio5 Institute, University of Arizona Genomics Core, Tucson AZ 85721, USA.

Received: 28 October 2013 Accepted: 13 December 2013 Published: 18 December 2013

\section{References}

1. Picard C, Fioramonti J, Francois A, Robinson T, Neant F, Matuchansky C: Review article: bifidobacteria as probiotic agents - physiological effects and clinical benefits. Aliment Pharmacol Ther 2005, 22:495-512.

2. Bottacini F, Milani C, Turroni F, Sanchez B, Foroni E, Duranti S, Serafini F, Viappiani A, Strati F, Ferrarini A, Delledonne M, Henrissat B, Coutinho P, Fitzgerald GF, Margolles A, van Sinderen D, Ventura M: Bifidobacterium asteroides PRL2011 genome analysis reveals clues for colonization of the insect Gut. PLoS One 2011, 7(9):e44229. doi:10.1371/journal.pone.0044229.

3. Anderson KE, Sheehan TH, Mott BM, Maes P, Snyder L, Schwan MR, Walton A, Jones BM, Corby-Harris V: Microbial ecology of the hive and pollination landscape: bacterial associates from floral nectar, the alimentary tract and stored food of honey bees (apis mellifera). PLoS One 2013. 8(12):e83125. doi:10.1371/journal.pone.0083125.

4. Martinson VG, Moy J, Moran NA: Establishment of characteristic gut bacteria during development of the honey bee worker. Appl Environ Microbiol 2012, 78:2830-2840.

5. Moran NA, Hansen AK, Powell JE, Sabree ZL: Distinctive Gut microbiota of honey bees assessed using deep sampling from individual worker bees. PLoS One 2012, 7:e36393. doi:10.1371/journal.pone.0036393.

6. Anderson KE, Sheehan TH, Eckholm BJ, Mott BM, DeGrandi-Hoffman G: An emerging paradigm of colony health: microbial balance of the honey bee and hive (Apis mellifera). Insect Soc 2011, 58:431-444.

7. Vojvodic S, Rehan SM, Anderson KE: Microbial Gut diversity of Africanized and European honey Bee larval instars. PLoS One 2013, 8(8):e72106. doi:10.1371/journal.pone.0072106.

8. Aziz RK, Bartels D, Best AA, DeJongh M, Disz T, Edwards RA, Formsma K, Gerdes S, Glass EM, Kubal M, Meyer F, Olsen GJ, Olson R, Osterman AL, Overbeek RA, McNeil LK, Paarmann D, Paczian T, Parrello B, Pusch GD, Reich C, Stevens R, Vassieva O, Vonstein V, Wilke A, Zagnitko O: The RAST server: rapid annotations using subsystems technology. BMC Genomics 2008, 8:9-75.

9. Gavini F, Van Esbroeck M, Touzel JP, Fourment A, Goossens H: Detection of fructose-6-phosphate phosphoketolase (F6PPK), a Key enzyme of the bifid-shunt, in Gardnerella vaginalis. Anaerobe 1996, 2:191-193.

10. Giraud M-F, Naismith JH: The rhamnose pathway. Curr Opin Struc Biol 2000, 10:687-696.

doi:10.1186/1757-4749-5-42

Cite this article as: Anderson et al:: Draft genome sequences of two Bifidobacterium sp. from the honey bee (Apis mellifera). Gut Pathogens 2013 5:42. 